\title{
Multi-mode Message Passing Switch Networks Applied for QC-LDPC Decoder
}

\author{
Chih-Hao Liu ${ }^{a}$, Chien-Ching Lin ${ }^{\mathrm{b}}$, Hsie-Chia Chang ${ }^{\mathrm{b}}$, Chen-Yi Lee ${ }^{\mathrm{b}}$ and Yarsun Hsu ${ }^{\mathrm{a}}$ \\ ${ }^{a}$ Department of Electrical Engineering, National Tsing-Hua University, \\ 101, Sec.2, Kuang-Fu Road, Hsinchu, Taiwan 30013, R.O.C. \\ b Department of Electronics Engineering, National Chiao-Tung University, \\ 1001, Ta-Hsueh Road, Hsinchu, Taiwan, R.O.C. \\ *Tel: +886-3-5131369*Email: jrhaulu@gmail.com
}

\begin{abstract}
The multi-mode message passing switch networks for multi-standard QC-LDPC decoder are presented. An enhanced self-routing switch network with only one barrel shifter permutation structure and a shifter-based two-way duplicated switch network are proposed to support 19 and 3 different submatrices defined in IEEE 802.16e and IEEE 802.11n. These proposed switch networks can route the decoding message in parallel by different sizes without signal congestion. The enhanced self-routing switch network can switch the messages at different expansion factors with the lowest hardware complexity. Under the condition of a smaller expansion factor, the decoder throughput can be enhanced from the two-way duplicated switch network by increasing the parallelism. In the $130 \mathrm{~nm}$ CMOS synthesis result, the proposed enhanced self-routing and the two-way duplicated switch network gate counts are $21.9 \mathrm{k}$ and $37.4 \mathrm{k}$ at $384 \mathrm{MHz}$ operation frequency.
\end{abstract}

Index Terms - LDPC code, decoder architectures, quasi-cyclic, IEEE 802.16e, WiMax, IEEE 802.11n, switch network.

\section{INTRODUCTION}

Low-density parity-check (LDPC) code, defined by a very sparse parity check matrix, was firstly introduced by Gallager [1]. The QC codes are described by sparse parity-check matrices comprised of blocks of circulant matrices [2]. The performance of the QC-LDPC block codes compares favorably with that of randomly constructed LDPC codes being shortened to medium block lengths [3]. The parity-check matrix $\mathbf{H}$ of QC-LDPC code can be decomposed into several cyclic-shifted identities or zero matrices. Message passing network at sub-matrix size is applied for QC-LDPC decoder to switch the decoding messages between the check nodes and the bit nodes [4]. In the other way, the QC-LDPC decoders are well suited for hardware implementation because of the regularity in the parity check matrices [5]. Newly high-speed communication systems such as IEEE 802.16e [6], IEEE 802.11n [7] have considered employing QC-LDPC codes to enhance their performance. But the multi-rate and multi-sizes QC-LDPC codes defined in IEEE 802.16e and IEEE 802.11n are irregular and difficult to support all code rates under variable matrix sizes [8].
According to different system parameters, the sub-matrix size defined by the expansion factor $z_{f}$ and the operation mode are variable in IEEE 802.16e and IEEE 802.11n.Dedicated hardware of message passing network for each specific submatrix size enhances the signal congestion with higher hardware complexity. It is necessary to have a flexible switch network to meet all sub-matrix sizes. The Omega and Benes sorting networks were exploited for regular LDPC decoders [4]. However, the traditional sorting networks, like Omega and Benes networks, are only limited to a fixed size and therefore suitable for the fixed size switching schemes.

With only one barrel shifter, the self-routing (SR) switch network was proposed to support 19 different sub-matrices sizes defined in IEEE 802.16e [9]. But additional control logic circuit is required to decide the expected routed messages when the shift amount is larger than half of maximum expansion factor. An enhanced SR switch network [10] can switch message in parallel with a simpler routing decision rule and reduce the control logic complexity. A shifter-based twoway duplicated switch network is proposed to upgrade the message passing throughput under the condition of a smaller sub-matrix size [11]. The selected results of duplicated switch network will be equivalent to the barrel shifting by shifting the duplicated parts with the original messages. These proposed switch networks can support 19 and 3 different sub-matrices defined in IEEE 802.16e and IEEE 802.11n.

The remainder of this paper is depicted as follows. Section II introduces IEEE 802.16e and IEEE 802.11n LDPC code structure. Section III describes the proposed architecture of the enhanced SR and two-way duplicated switch network which can support all the sub-matrix sizes defined in IEEE 802.16e and IEEE $802.11 \mathrm{n}$. Finally, the implementation results of the proposed switch networks applied for the QC-LDPC decoder are shown in section IV and the final conclusion is presented in section $\mathrm{V}$.

\section{QC-LDPC CODES IN IEEE 802.16E/IEEE 802.11N}

In the IEEE 802.16e mobile WiMax and IEEE 802.11n wireless LAN systems, the $M \times N$ parity-check matrix $\mathrm{H}$ can be decomposed into $z_{f} \times z_{f}$ sub-matrices, where the variable $M$ is the number of parity check equations, and $N$ is the code length. Each sub-matrix is either the zero matrix or the cyclic- 
shifted identity matrix. Note that the expansion factor $z_{f}$, has 19 in IEEE 802.16e [6] and 3 in IEEE 802.11n [7]. The paritycheck matrix $\mathbf{H}$ can also be expanded from the $m_{b} \times n_{b}$ base matrix $\mathbf{H}_{\mathbf{b}}$ with $m_{b}=M / z_{f}$ and $n_{b}=N / z_{f}$. The code rate is determined by the value of $m_{b} / n_{b}$, where the maximum value of $m_{b}$ and the constant value of $n_{b}$ defined in the IEEE 802.16e system are 12 and 24 respectively. The parity check matrix size is determined based on the code rate and the expansion factor $z_{f}$ of sub-matrices.

Table I shows the system parameters of IEEE 802.16e and IEEE $802.11 \mathrm{n}$. The 19 variable expansion factors in the IEEE 802.16e specification [6] range from 24 to 96 with an increment of four, and the 3 variable expansion factors in the IEEE 802.11n specification [7] range from 27 to 81 with an increment of 21. The $\mathbf{H}$ is extended from the base matrix $\mathbf{H}_{\mathrm{b}}$ by replacing each 1 in $\mathbf{H}_{\mathrm{b}}$ with a $z_{f} \times z_{f}$ circular right shifted identity matrix and each 0 in $\mathbf{H}_{\mathrm{b}}$ with a $z_{f} \times z_{f}$ zero matrix. A structure for rate-1/2 parity check matrix $\mathbf{H}$ is shown in Fig. 1. Note that $\mathbf{H}$ can be partitioned into two parts: $\mathbf{H}_{\mathbf{1}}$ and $\mathbf{H}_{2}$, where $\mathbf{H}_{1}$ is the information bits and $\mathbf{H}_{2}$ is the parity check bits. $\mathbf{H}_{\mathbf{2}}$ can also be partitioned into two parts: $\mathbf{h}$ and $\mathbf{H}_{2}{ }_{2}$, where $\mathbf{H}_{2}$ has a dual-diagonal structure.

TABLE I. SYSTEM PARAMETERS OF IEEE 802.16E AND IEEE $802.11 \mathrm{~N}$

\begin{tabular}{|c|c|c|c|c|}
\hline & Code Rate & Expansion Factor $z_{f}$ & Block Length $N$ & Throughput \\
\hline IEEE 80216e & $\begin{array}{c}1 / 2,2 / 3 \mathrm{~A}, 2 / 3 \mathrm{~B}, \\
3 / 4 \mathrm{~A}, 3 / 4 \mathrm{~B}, 5 / 6\end{array}$ & $\begin{array}{c}24+4 K \\
\mathrm{~K}=[0,1,2 \cdots 18]\end{array}$ & $\begin{array}{c}576+96 K \\
\mathrm{~K}=[0,1,2 \cdots 18\end{array}$ & $30 \mathrm{Mb} / \mathrm{s}$ \\
\hline IFEE 802.11n & $1 / 2,2 / 3,3 / 4,5 / 6$ & $27,54,81$ & $648,1296,1944$ & $300 \mathrm{Mb} / \mathrm{s}$ \\
\hline
\end{tabular}

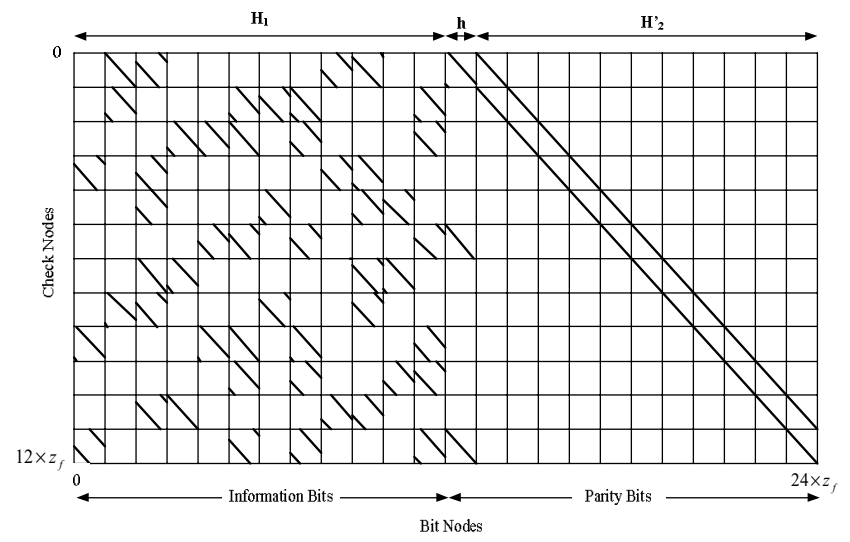

Figure 1. Structure of The Parity Check Matrix for a Rate 1/2 IEEE 802.16e LDPC Code

\section{MUTIL-MODE MESSAGE PASSING SWITCH NETWORK}

The variable sub-matrix size causes the difficulty in applying the fixed size crossbar switches, such as Omega and Benes sorting networks [4]. For multi-standard QC-LDPC decoder, two shifter-based structures with one single permutation network [10][11] are proposed to complete the message routing for all code rates and lengths. The enhanced SR switch network is suitable for message passing at the larger sub-matrix size, and meets all sub-matrix sizes with a simpler routing decision rule. Under the condition of a smaller sub-matrix size, the two-way duplicated switch network can be configurable for different expansion factors and switch different messages concurrently to improve the decoding throughput.

\section{A. Enhanced Self-routing Switch Network}

With only one barrel shifter, the SR switch network was proposed to support 19 different sub-matrices sizes defined in IEEE 802.16e [9]. Fig.2 illustrates the SR switch network that routes all messages concurrently through the three-stage switch network. The first stage is the combination of source messages with the self-routing bits (SRB), the second stage is the barrel shifter permutation network, and the third stage is the selection scheme. During the final selection, the expected $z$ (smaller than the maximum expansion factor $z_{\text {max }}$ ) output messages are chosen from the $z_{\max }$ routed messages according to the SRBs determined at the first stage. Note that the variable $z$ is the expected expansion factor.

The third stage routing lookup engine compares the corresponding routed self-routing bits in parallel according to the expected expansion factor and shift amount. The routed messages that the available corresponding self-routing (selfrouting bit $=1$ implies the available condition) are selected as the expected output messages. But both the routed SRBs for the routing lookup engine are available when the shift amount is larger than $z_{\max } / 2$. An additional logic circuit is needed to decide the expected output message in the SR switch network. With a simpler routing decision rule, the enhanced SR network [11] is proposed to route the message with the lower hardware complexity.

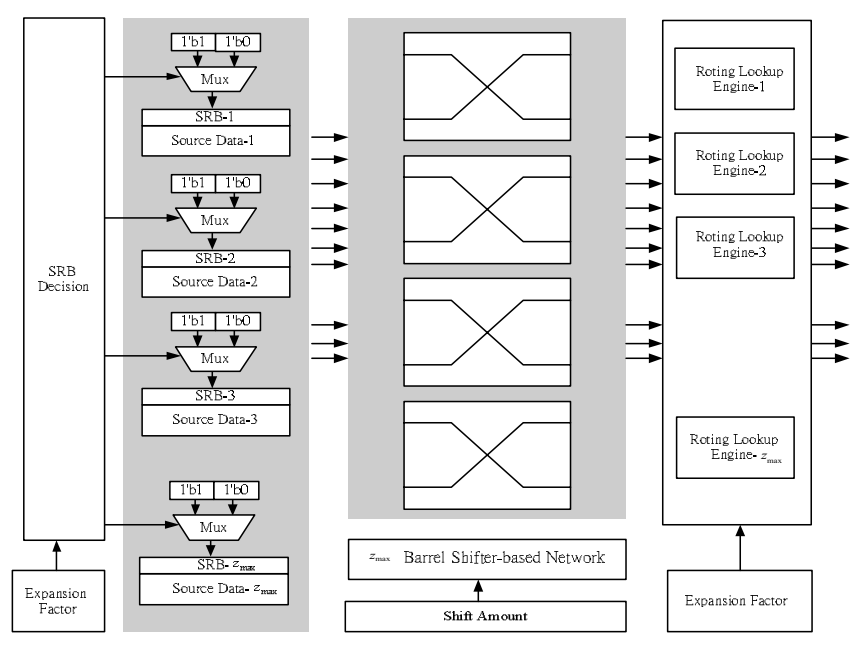

Figure 2. Self-routing Switch Network Architecture

Fig. 3(a) shows that the first routing decision data are constructed from the $(z+1)$-th to the $2 z$-th routed data and the second routing decision data are constructed from the first to the $z$-th routed data. Note that the expected expansion factor $z$ $\leq z_{\max } / 2$ in Fig. 3(a). Fig. 3(b) shows the routing lookup scheme for $z>z_{\max } / 2$. The first part of first routing decision data are constructed from the $(z+1)$-th to the $z_{\max }$-th routed data and the second part of first routing decision data are constructed from the first to the $(2 z-96)$-th routed data. The second routing decision data are constructed from the first to 
the $z$-th routed data. When the shift amount is larger than $z_{\max } / 2$ and both the compared corresponding routed SRBs are available, the first routing decision data will be always chosen as the output message.

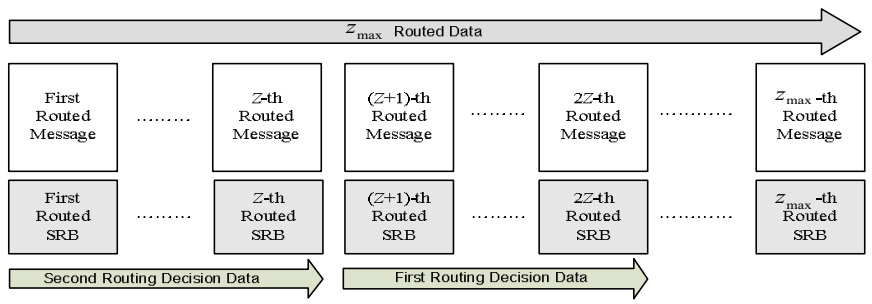

(a) The Routing Decision Data for Smaller Expected Expansion Factor

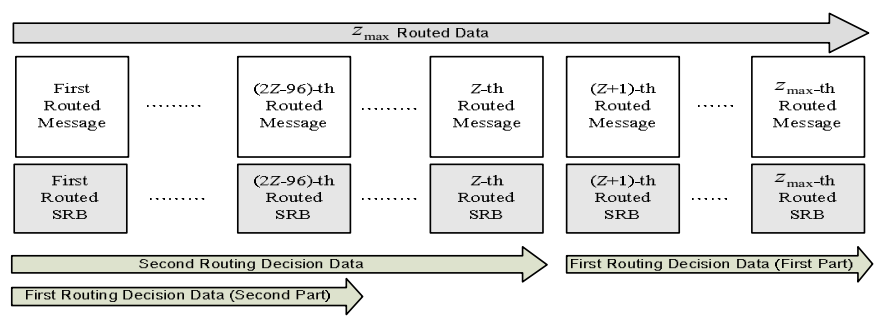

(b) The Routing Decision Data for Expected Expansion Factor

Figure 3. The Enhanced SR Routing Lookup Scheme

\section{B. Two-way Duplicated Switch Netwok}

We proposed the shifter-based two-way duplicated switch network to route the messages associated with different submatrices concurrently [11]. The proposed switch network duplicates the original messages, and switches different size messages by the shifter circuits concurrently. Consequently, the selected results will be equivalent to the barrel shifting.

The two-way duplicated switch network can improve the decoding efficiency when the expected expansion factor $z$ is smaller than $z_{\max } / 2$. By a smaller expansion factor, the switch network can be configured to process two-way duplicated messages associated with different sub-matrices. Fig. 4 shows the source message format of two-way duplicated parallelism. By transferring the original message to the source message format, the first and the second message can be switched simultaneously according to the different shift amounts.

\begin{tabular}{|c|c|c|c|c|c|}
\hline $\begin{array}{c}\text { First Original } \\
\text { Message } \\
\text { First }(z-1) \text { th }\end{array}$ & $\begin{array}{c}\text { First Duplicated } \\
\text { Message } \\
\text { First-(z-1)th }\end{array}$ & Dummy & $\begin{array}{c}\text { Second Original } \\
\text { Message } \\
\text { First-(z-1)th }\end{array}$ & $\begin{array}{c}\text { Second Duplicated } \\
\text { Mes sage } \\
\text { First }(z-1) \text { th }\end{array}$ & Dummy \\
\hline
\end{tabular}

Figure 4. The Source Message of Proposed Two-way Duplicated Switch Network

Fig. 5 illustrates the two-way duplicated switch network architecture, where $2 z_{\max }$ messages are routed in parallel through the proposed three-stage switch network. The message exchange operations are as follows: the first stage will merge the original and the duplicated message, and generate the source message according to the expansion factor and the parallelism mode. The second stage is the shifter network composed of two $z_{\max }$-size and one $2 z_{\max }$-size shifters. Note that the maximum supporting shift amount supported by the shifter is $z_{\max }-1$. The third stage is the output message selector. Output message controller will select the expected switched message from the $2 z_{\max }$ routed messages.

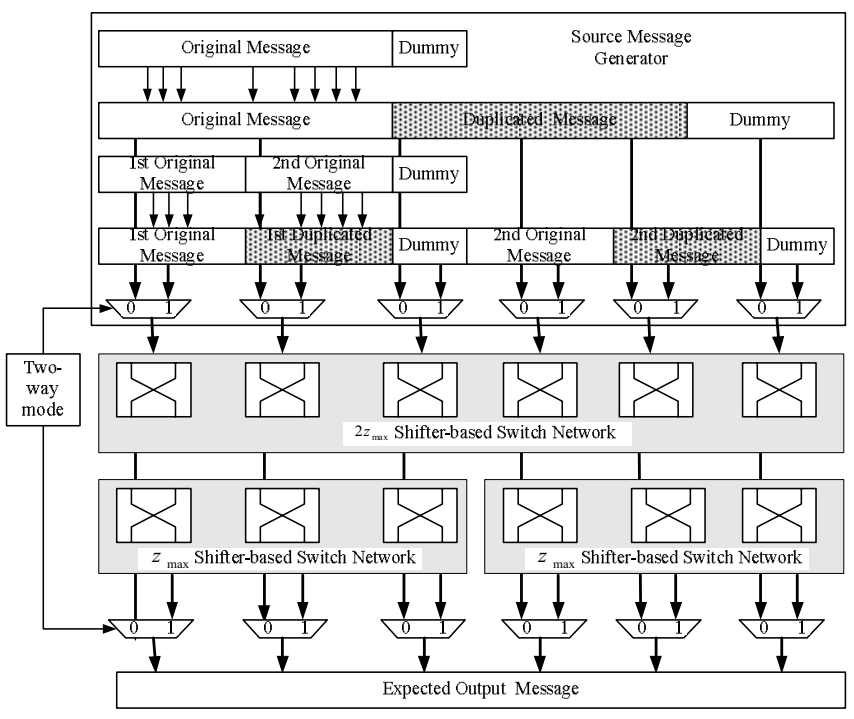

Figure 5. The Architecture of Two-way Duplicated Switch Network

\section{Chip IMPLEMENTATION RESUlT}

Table II is a summarized performance comparison among the proposed switch networks [10][11] and the existing switch network. In the $130 \mathrm{~nm}$ CMOS synthesis result, the proposed enhanced SR and the two-way duplicated switch network occupy $0.1095 \mathrm{~mm}^{2}$ and $0.1872 \mathrm{~mm}^{2}$ area at $384 \mathrm{MHz}$ operation frequency respectively. The flexible permuter proposed in [12] can support three expansion factors defined in IEEE $802.11 \mathrm{n}$ only. The flexible barrel shifter and the multi-stage shifting network with multi-stage multiplexers were applied to switch variable size messages for IEEE 802.16e LDPC decoders [13][14][15]. But these switch networks increase the signal congestion since the duplicated multiplexers for the variable sub-matrix sizes. With the single permutation network, the proposed enhanced SR switch network can meet all the expansion factor requirements of IEEE 802.16e and IEEE 802.11n with the lowest hardware complexity. To improve the throughput when $z \leq z_{\max } / 2$, the two-way duplicated switch network can switch two messages groups simultaneously.

The two-way duplicated switch network was applied to the QC-LDPC decoder, compliant to both IEEE 802.11n and IEEE 802.16e. The message passing efficiency is double as compared to the enhanced-SR switch network if $z_{f}<z_{\max } / 2$. In the $90 \mathrm{~nm} 1 \mathrm{P} 9 \mathrm{M}$ process, the decoder chip occupies $6.25 \mathrm{~mm}^{2}$ silicon area and can achieve $300 \mathrm{MHz}$ in the post-layout simulation. For the IEEE 802.16e application, the decoder equipped with two-way duplicated switch network chip operating at $107 \mathrm{MHz}$ frequency achieves the maximum 63.36 $\mathrm{Mb} / \mathrm{s}$ data rate within 20 iterations and dissipates $203 \mathrm{~mW}$ at $1.0 \mathrm{~V}$ supply. 
TABLE II. COMPARISON OF SWITCH NETWORK FOR LDPC DECODER

\begin{tabular}{|c|c|c|c|c|c|}
\hline & $\begin{array}{c}\text { Our Enhanced SR } \\
\text { Switch Network } \\
{[10]}\end{array}$ & $\begin{array}{c}\text { Our Two-way } \\
\text { Duplicated Switch } \\
\text { Network [1 } 1]\end{array}$ & $\begin{array}{c}\text { Flexible } \\
\text { Permuter [12] }\end{array}$ & \begin{tabular}{|c} 
Flexible \\
Barrel Shifter [13]
\end{tabular} & $\begin{array}{c}\text { Multi-size } \\
\text { Shifting Network } \\
{[14][15]}\end{array}$ \\
\hline $\begin{array}{c}\text { Message Word } \\
\text { Length } \\
\end{array}$ & 6-bit & 6-bit & 8-bit & 9-bit & 6-bit \\
\hline Process & UMC 130nm & UMC $130 \mathrm{~nm}$ & $130 \mathrm{~nm}$ & STM 130nm & $130 \mathrm{~nm}$ \\
\hline $\begin{array}{l}\text { Implemetation } \\
\text { Area }\left(\mathrm{mm}^{2}\right)\end{array}$ & $\begin{array}{l}0.1095 \\
\text { (a) } 384 \mathrm{MHz}\end{array}$ & $\begin{array}{c}0.1872 \\
\text { (a) } 384 \mathrm{MHz}\end{array}$ & (a) $\overline{412 \mathrm{MHz}}$ & $\begin{array}{c}0.511 \\
\text { (a) } 333 \mathrm{MHz}\end{array}$ & $\begin{array}{c}0.2776 \\
\text { (a) } 500 \mathrm{MHz}\end{array}$ \\
\hline Gate Counts & $21.9 \mathrm{k}$ & $37.4 \mathrm{k}$ & $23.1 \mathrm{k}$ & 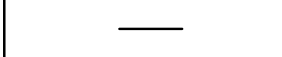 & $\longrightarrow$ \\
\hline $\begin{array}{l}\text { Compliant to } \\
\text { IEEE 802.16e }\end{array}$ & Yes & Yes & No & Yes & Yes \\
\hline $\begin{array}{l}\text { Compliant to } \\
\text { IEEE 802.1 ln }\end{array}$ & Yes & Yes & Yes & No & No \\
\hline$\underset{z_{f} \leq 48}{\text { Parallelism with }}$ & One & Two & One & One & One \\
\hline
\end{tabular}

\section{CONCLUSION}

The enhanced SR switch network and the two-way duplicated switch network can support the permutation function that fulfills the requirement of different sub-matrix sizes. Signal routing congestion in the proposed switch networks can significantly be reduced with only one permutation shifter that provides $19+3$ different switch network sizes defined in the IEEE 802.16e and the IEEE $802.11 \mathrm{n}$. After synthesized by the $130 \mathrm{~nm}$ process, the enhanced SR switch network can switch messages at different sub-matrix sizes with the lowest hardware complexity. Under a smaller expansion factor, the two-way duplicated switch network can result in 50\% improvement in decoding throughput by increasing the parallelism of message passing.

\section{REFERENCES}

[1] R. Gallager, "Low-density parity-check codes," Information Theory, IEEE Transactions on, vol. 8, pp. 21-28, Jan. 1962.

[2] M. P. C. Fossorier, "Quasi-cyclic low-density parity-check codes from circulant permutation matrices," IEEE Trans. on Info. Theory, vol. 50, pp. 1788 - 1793, Aug. 2004.

[3] L. Chen, J. Xu, I. Djurdjevic and S. Lin, "Near-Shannon-Limit QuasiCyclic Low-Density Parity-Check Codes," IEEE Trans. on Communications, vol. 52, pp. 1038 -1042, Jul. 2004.

[4] M. M. Mansour and N. R. Shanbhag, "A 640-Mb/s 2048-Bit Programmable LDPC Decoder Chip," IEEE J. of Solid-State Circuits, vol. 41, no. 3, pp.634 -698, Mar. 2006.

[5] Zhongfeng Wang and Zhiqiang Cui, "Low-Complexity High-Speed Decoder Design for Quasi-Cyclic LDPC Codes," IEEE Trans. on Very Large Scale Integr.(VLSI) Syst. vol. 15, no. 1, pp.104-114, Jan. 2007.
[6] "Part 16: air interface for fixed and mobile broadband wireless access systems amendment for physical and medium access control layers for combined fixed and mobile operation in licensed bands," IEEE P802.16e-2005, Oct. 2005.

[7] IEEE 802.11 Wireless LANs WWiSE Proposal: High Throughput extension to the 802.11 Standard. IEEE 11-04-0886-00-000n.

[8] X. Y. Shih, C. Z. Zhan, C. H. Lin, and A. Y. Wu, "A 19-mode $8.29 \mathrm{~mm}$ 52-mW LDPC Decoder Chip for IEEE 802.16e System," IEEE Symp.VLSI Circuits and VLSI Technology, pp. 16-17, June 2007.

[9] C. H. Liu, S. W. Yen, C. L. Chen, H. C. Chang, C. Y. Lee, Y. S. Hsu, and S. J. Jou, "An LDPC Decoder Chip Based on Self-Routing Network for IEEE 802.16e Applications," to apear in the IEEE J. of Solid-State Circuits.

[10] C. H. Liu, C. C. Lin, H. C. Chang, C. Y. Lee, and Y. S. Hsu, "Method and Apparatus for Switching Data in Communication Systems," Taiwan and US patent pending.

[11] C. H. Liu , C. C. Lin , H. C. Chang, C. Y. Lee, and Y. S. Hsu,"Method and Apparatus for Muti-mode and $m$-way Parallel Data Switch in Communication Systems," Taiwan, Japan and US patent pending.

[12] M. Karkooti, P. Radosavljevic, and J. R. Cavallaro, "Configurable, High Throughput, Irregular LDPC Decoder Architecture: Tradeoff Analysis and Implementation," Proc. of 17th International Conference on Application-specific Systems and Processors (ASAP'06), pp. $360-$ 367, Sept. 2006.

[13] T. Brack, M. Alles, F. Kienle, and N. When, "A Synthesizable IP Core for WIMAX 802.16E LDPC Code Decoding," IEEE 17th Interantional Symposium on Personal, Indoor and Mobile Radio Communications (PIMRC'06), pp. 1 -5, Sept. 2006.

[14] M. Rovini, N. E. L'Insalata, F. Rossi, and L. Fanucci, "A 565 Mbps Multi-Rate IP-core Decoder of Structured LDPC Codes", ST J. Research Multimedia Streaming Technologies, vol. 3, no. 2, Dec. 2006.

[15] M. Rovini, G. Gentile, and L. Fanucci, "Multi-size circular shifting networks for decoders of structured LDPC codes," Electronics Letters, vol 43, Issue 17, pp. 938 - 940, Aug. 2007. 